\title{
Zeit für dich - Zeit für mich - Zeit für uns
}

\author{
Hans Kurt \\ Dr. med., FMH-Vertreter im Zentralkomitee «Tag der Kranken»
}

\begin{abstract}
Wir alle - nicht nur Ärzte* - stehen unter Zeitdruck. Rasch soll es gehen, effizient muss es sein und unser Handeln muss optimiert werden. Die Zeit ist dabei ein wesentlicher Faktor. Deshalb ist der Tag der Kranken dieses Jahr diesem wichtigen Thema gewidmet.
\end{abstract}

Die Medizin gleicht mehr und mehr einem Produktionsprozess. "Je mehr Medizin als Produktionsprozess betrachtet wird, desto mehr werden Aktionismus befördert, das Machen belohnt, das Zuhören bestraft, die Interventionszeit berechnet, die Beratungszeit übersehen, die Steigerung des Durchlaufs zum Wert erhoben und die Behutsamkeit und Sorgfalt als etwas angesehen, was den Betrieb nur aufhält», stellt Prof. Dr. med. Giovanni Maio aus Freiburg i. Br. in einem Artikel der «Synapse» fest.

Untersuchungen zu Patienten-Arzt-Interaktionen zeigen, dass der einleitende Bericht des Patienten über seine Beschwerden durch den Arzt häufig bereits zu einem sehr frühen Zeitpunkt unterbrochen wird, nämlich nach 10 bis 20 Sekunden, bevor der Patient überhaupt Zeit hat, sein Anliegen so zu schildern, dass er sich verstanden fühlt, und der Arzt überhaupt verstehen kann, um was es schlussendlich geht. Würden Patienten nicht unterbrochen, würden sie ganze 90 Sekunden sprechen. Sich Zeit nehmen oder eben Zeit einsparen sind wesentliche Faktoren im Alltag von Kranken, ihren Angehörigen, Ärzten und Pflegenden. Im Rahmen der Ökonomisierung des Gesundheitswesens, des Primates, dass jede unserer Handlungen und deren Resultate in Zahlen abbildbar sein muss, spielt der Faktor Zeit eine wesentliche Rolle, lässt sich doch Zeit messen und erfassen. Wir kennen sie, die Worte Zeiterfassung, Zeittarif, Eingriffszeit, Aufenthaltsdauer, Prozessdauer. Und so tickt die Uhr auch in der Medizin schneller und schneller.

\section{Weniger Zeit für patientennahe Tätigkeiten}

Die durchschnittliche Aufenthaltsdauer in Schweizer Spitälern und Kliniken ist in den letzten Jahren kontinuierlich gesunken. Trotzdem sind die Spitäler weiterhin gut belegt. In verschiedenen Studien, u.a. am
Universitätsspital CHUV in Lausanne und am Kantonsspital Baden, wird gezeigt, dass Assistenzärzte täglich 100 respektive 90 Minuten direkt am Bett des Patienten verbringen, was einem direkten Patientenkontakt von gerade mal ca. 15 Minuten entspricht und dies bei einer durchschnittlichen täglichen Arbeitszeit von 11,5 Stunden. Dreimal mehr Zeit verbringen Ärzte vor dem Computer. Laut einer Studie der FMH nehmen die patientennahen Tätigkeiten in der Akutsomatik und in der Psychiatrie nur noch rund ein Drittel der Arbeitszeit ein. In der ambulanten Praxis nimmt der Administrationsaufwand ebenfalls zu. Nach dem Tarifeingriff des Bundesrates gilt es heute, Leistungen im Minutentakt zu erfassen.

In den letzten Jahren ist der Zusammenhang zwischen Krankheit und Arbeitsfähigkeit zu einem allumfassenden Thema in unserer Gesellschaft geworden, ist doch der arbeitende Mensch der Beweis für Gesundheit, Anerkennung und Lebenssinn. Wir sprechen viel über die Integration in die Arbeitswelt. Und die Politik hat es sich zum Ziel gesetzt, Versicherungsmissbrauch zu entlarven und den Patienten möglichst rasch wieder an der Arbeit zu sehen. Vergessen wird dabei, dass viele unserer Patienten aus Pflichtgefühl und Angst, die

\section{Verein «Tag der Kranken»}

Der Verein "Tag der Kranken» ist eine gemeinnützige Organisation, zusammengesetzt aus Patientenorganisationen, Gesundheitsligen, Fachverbänden, der GDK und auch der FMH. Er wurde 1939 gegründet und sensibilisiert die Bevölkerung einmal pro Jahr zu einem besonderen Thema. DerTag der Kranken soll dazu beitragen, die Beziehung zwischen Kranken und Gesunden zu fördern, Mitgefühl zu zeigen und das Verständnis für die Bedürfnisse von Kranken und Angehörigen zu schaffen. Dieses Jahr findet derTag der Kranken am 4. März statt und bietet Anlass für verschiedenste Veranstaltungen in der Öffentlichkeit.

Weitere Informationen: www.tagderkranken.ch 
Arbeit zu verlieren, weiterarbeiten - selbst wenn sie eigentlich krank sind. Aus einer Studie von Travail. Suisse geht hervor, dass der Präsentismus in der Schweiz weit verbreitet ist. 30 Prozent der repräsentativ Befragten geben an, dass sie oft oder häufig arbeiten, obwohl sie krank sind. Es versteht sich von selbst, dass so die Zeit für das Auskurieren einer Krankheit oft fehlt. Der problematische Einsatz von Medikamenten, insbesondere von Analgetika und gewissen Psychopharmaka, lässt uns zwar vermeintlich Zeit sparen, verursacht aber neue, andere Probleme und Schwierigkeiten.

Zeit haben, krank zu sein, ist das eine. Zeit haben, um kranke Kinder oder ältere Menschen zu betreuen, das andere. Gemäss Zahlen des Bundesamtes für Statistik leistet ein Drittel der Schweizer Bevölkerung ab 15 Jahren informelle Freiwilligenarbeit. Nachbarschaftshilfe, Kinderbetreuung sowie Dienstleistung in der Pflege von Verwandten und Bekannten ergeben jährlich ca. 64 Millionen Pflege- und Betreuungsstunden in einem Gegenwert von 3,5 Milliarden Franken. Die kommenden Jahre werden mit der Zunahme an älteren Menschen diese Unterstützungsform noch ansteigen lassen. Angehörige haben, um ihre Aufgaben zu erfüllen, auch Wünsche an die Ärzteschaft und die Pflegenden. Bei einer Befragung von Angehörigen von psychisch kranken Menschen über ihre Wünsche an die Psychiatrie war mit 81 Prozent der grösste Wunsch der, dass mehr Zeit für Gespräche mit den Angehörigen zur Verfügung stehen sollte. Angehörige brauchen Zeit für Informationen, für Gespräche und eine respektvolle Anerkennung ihrer Betreuungsarbeit. Und Angehörige brauchen auch Zeit für die eigene Erholung und ihre eigenen Bedürfnisse.

Korrespondenz:

Dr. med. Hans Kurt

Facharzt für Psychiatrie

und Psychotherapie

Bielstrasse 109

4500 Solothurn

kurt[at]solnet.ch

\section{Zeit ist wesentlich für gelingende Behandlung}

Zeit haben oder nicht beeinflusst wesentlich unsere ärztliche Tätigkeit und die Medizin ganz generell. Aus

\section{Ursula Steiner-König}

Ende August 2017 ist Frau Dr. med. Ursula Steiner-König im Alter von 78 Jahren in Basel verstorben. Frau Dr. Steiner war, delegiert durch die FMH, lange Jahre Vizepräsidentin des Zentralkomitees "Tag der Kranken». Durch ihre verschiedenen politischen Engagements, u.a. als Vize-Präsidentin der FMH, hat sie ihre grossen $\mathrm{Er}$ fahrungen engagiert und mit viel Fachwissen für den «Tag der Kranken» eingesetzt. Dafür sei ihr im Gedenken herzlich gedankt.

Befragungen geht hervor, dass Ärzte, die genügend Zeit für Gespräche mit Patienten und Angehörigen haben, ihre Arbeit mit mehr Zufriedenheit erleben. Die Beziehung zwischen Arzt und Patient wird wieder zur Basis des ärztlichen Handelns, Zeit nehmen und geben zu einem wesentlichen Erfolgsfaktor für eine gelingende Behandlung. Nehmen wir uns also Zeit für unsere $\mathrm{Pa}$ tienten, für deren Angehörige, aber auch für unsere Umgebung, sei es während der Arbeit, sei es im privaten Leben. Machen wir wieder einmal eine ausgiebige Kaffeepause mit den Praxisassistentinnen. Plaudern wir wieder einmal mit den Pflegenden im Abteilungsbüro. Erzählen wir von unseren Schwierigkeiten im beruflichen Alltag, aber auch von guten Begegnungen und dankbaren Patienten. Nehmen wir uns auch im hektischen Alltag einmal Zeit, legen die Beine auf das Pult, schauen zum Fenster hinaus und geniessen für einen kurzen Moment das vorbeiziehende Wetter. Und wenn wir dann nach einem zeitintensiven Arbeitstag nach Hause zurückkehren, sollten wir uns vielleicht nicht als erstes in medizinischer Fachliteratur vertiefen, sondern sich mit der Familie an den Tisch setzen, wieder einmal ein Kartenspiel hervornehmen, später in aller Ruhe einen Tee trinken und uns genügend Zeit für Ruhe und Erholung gönnen.

In dem Sinne ist das Thema des diesjährigen Tag der Kranken nicht nur ein Thema für den Kranken, sondern ein Thema für uns alle, ganz speziell auch für uns Ärzte, die so oft glauben, keine Zeit zu haben. 